\title{
Distanciamento social, sentimento de tristeza e estilos de vida da população brasileira durante a pandemia de Covid-19
}

\author{
Social distancing, feeling of sadness and lifestyles of the Brazilian \\ population during the Covid-19 pandemic
}

\author{
Deborah Carvalho Malta', Crizian Saar Gomes ${ }^{\mathbf{1}}$, Célia Landmann Szwarcwald ${ }^{\mathbf{2}}$, Marilisa Berti

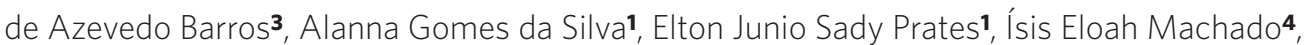 \\ Paulo Roberto Borges de Souza Júnior ${ }^{2}$, Dália Elena Romero², Margareth Guimaraes Lima ${ }^{3}$, Giseli \\ Nogueira Damacena ${ }^{2}$, Luiz Otávio Azevedo ${ }^{2}$, Maria de Fátima Pina ${ }^{2}$, André Oliveira Werneck ${ }^{\mathbf{5}}$, \\ Danilo Rodrigues Pereira da Silva 6
}

DOI: $10.1590 / 0103-11042020 E 411$

'Universidade Federal de Minas Gerais (UFMG)

- Belo Horizonte (MG), Brasil.

dcmalta@uol.com.br

2 Fundação Oswaldo Cruz (Fiocruz), Instituto de

Comunicação e Informação Científica e Tecnológica em Saúde (Icict) - Rio de Janeiro (RJ), Brasil.

3 Universidade Estadual de Campinas (Unicamp) Campinas (SP), Brasil.

4 Universidade Federal de Ouro Preto (Ufop) - Ouro Preto (MG), Brasil.

5 Universidade de São Paulo (USP) - São Paulo (SP), Brasil.

6 Universidade Federal de Sergipe (UFS) - Aracajú (SE), Brasil.
RESUMO O objetivo do estudo foi analisar a adesão ao distanciamento social, as repercussões no estado de ânimo e as mudanças nos estilos de vida da população adulta brasileira durante o início da pandemia da Covid-19. Estudo transversal com indivíduos adultos residentes no Brasil ( $\mathrm{n}=45.161$ ) que participaram do inquérito de saúde virtual ConVid - Pesquisa de Comportamentos, no período de 24 de abril a 24 de maio de 2020. Da amostra estudada, apenas 1,5\% levou vida normal, sem nenhuma restrição social, e $75 \%$ ficaram em casa, sendo que, destes, $15 \%$ ficaram rigorosamente em casa. Os sentimentos frequentes de tristeza ou depressão (35,5\%), isolamento (41,2\%) e ansiedade (41,3\%) foram reportados por grande parte da população estudada. Verificou-se que $17 \%$ dos participantes reportaram aumento do consumo de bebidas alcoólicas e que $34 \%$ dos fumantes aumentaram o número de cigarros. Observou-se aumento no consumo de alimentos não saudáveis e redução da prática de atividade física no período estudado. Conclui-se que houve elevada adesão ao distanciamento social e aumento dos sentimentos de tristeza, depressão e ansiedade, bem como aumento de consumo de alimentos não saudáveis, uso de bebidas alcóolicas e cigarros e redução da prática de atividade física.

PALAVRAS-CHAVE Quarentena. Infecções por coronavírus. Saúde mental. Estilo de vida. Brasil.

\begin{abstract}
The aim was to analyze adherence to social distancing, repercussions on the mood and changes in the lifestyles of the Brazilian adult population during the beginning of the Covid-19 pandemic. A crosssectional study with adult individuals living in Brazil $(n=45,161)$ who participated in the virtual health survey ConVid - Behavior Survey, from April 24 to May 24, 2020. Of the sample studied, only 1.5\% led normal lives, without any social restrictions and 75\% stayed at home, of which 15\% stayed strictly at home. The frequent feelings of sadness or depression (35.5\%), isolation (41.2\%) and anxiety (41.3\%) were reported by a large part of the studied population. It was found that $17 \%$ of participants reported increased consumption of alcoholic beverages and $34 \%$ of smokers increased the number of cigarettes. There was an increase in the consumption of unhealthy foods and a reduction in the practice of physical activity in the period studied. It was concluded that there was a high adherence to social distancing and an increase in feelings of sadness, depression and anxiety, as well as an increase in the consumption of unhealthy foods, use of alcoholic beverages and cigarettes and a reduction in the practice of physical activity.
\end{abstract}

KEYWORDS Quarantine. Coronavirus infections. Mental health. Life style. Brazil. 


\section{Introdução}

A pandemia da doença causada pelo coronavírus 2019 (Covid-19) foi reconhecida pela Organização Mundial da Saúde (OMS) em 11 de março de 2020'. No Brasil, o primeiro caso confirmado foi no estado de São Paulo, no dia 26 fevereiro ${ }^{2}$. Até o dia 24 junho, 1.145.906 casos foram confirmados, e 52.645 óbitos atestados, revelando uma letalidade no País de 4,9\% ${ }^{3}$.

Em função da inexistência de medidas preventivas, a OMS recomendou a adoção de intervenções não farmacológicas, entre elas, o distanciamento social, com o objetivo de diminuir o contato físico entre pessoas e o risco de transmissão da Covid-19, bem como de promover o achatamento da curva de crescimento dos casos ${ }^{4}$. As primeiras medidas foram adotadas na China, onde mais de um terço da população chegou a ficar em isolamento social ${ }^{5,6}$.

No Brasil, diversas medidas de distanciamento social foram adotadas pelos estados e municípios, como fechamento de escolas e comércios não essenciais, restrição na circulação de ônibus, incentivo ao trabalho em casa, e fechamento de cidades e estados mais afetados 7,8 .

O distanciamento social é a medida mais eficaz para prevenção da Covid-199,10, contudo, tem potenciais repercussões clínicas e comportamentais, podendo resultar em adoecimento psíquico e em mudanças nos estilos de vida, como a redução da prática de atividade física, aumento do estresse e do consumo de bebidas alcoólicas, tabaco e de alimentos não saudáveis ${ }^{11-16}$. Os efeitos negativos associados do distanciamento social poderão ser observados em curto, médio e longo prazo na saúde individual e coletiva.

Destaca-se, ainda, que a pandemia da Covid-19 aumentou a situação de extrema vulnerabilidade da população brasileira, com altas taxas de desemprego e redução de renda17. Além disso, políticas de austeridade previamente adotadas podem comprometer as respostas do Sistema Único de Saúde (SUS) no atendimento ${ }^{18-20} \mathrm{e}$ no enfrentamento da Covid-19, e das Doenças Crônicas Não Transmissíveis (DCNT) ${ }^{21}$. Assim, torna-se imperativo analisar o comportamento das situações de saúde da população brasileira, durante a pandemia visando apoiar as respostas do setor de saúde.

Nesse sentido, o objetivo deste estudo foi analisar a adesão ao distanciamento social, as repercussões no estado de ânimo e as mudanças nos estilos de vida da população adulta brasileira durante a pandemia da Covid-19.

\section{Material e métodos}

Trata-se de um estudo transversal realizado com indivíduos adultos ( $\geq 18$ anos) residentes no Brasil que participaram do inquérito de saúde virtual ConVid - Pesquisa de Comportamentos. A coleta de dados do ConVid foi via web, utilizando-se de um questionário de autopreenchimento por meio de celular ou computador com acesso à internet. O questionário foi construído por intermédio do aplicativo RedCap (Research Eletronic Data Capture), uma plataforma para coleta, gerenciamento e disseminação de dados de pesquisas. As informações foram coletadas diretamente pela internet e armazenadas no servidor do Instituto de Comunicação e Informação Científica e Tecnológica em Saúde da Fundação Oswaldo Cruz (Icict/Fiocruz).

O convite aos participantes foi feito por um procedimento de amostragem em cadeia, chamado, bola de neve virtual ${ }^{22}$. Inicialmente, os pesquisadores do estudo escolheram um total de 200 outros pesquisadores de diferentes estados do Brasil. Adicionalmente, cada pesquisador do estudo selecionou 20 pessoas da sua rede social. As pessoas escolhidas na primeira etapa foram chamadas de sementes (influenciadores) porque foram elas que desencadearam a rede de convidados. Após responder ao questionário, as sementes constituíram a primeira onda da cadeia de recrutamento. Por sua vez, as sementes enviaram o link da pesquisa para, pelo menos, 12 pessoas das suas redes sociais, obedecendo a uma estratificação por sexo, faixa de idade (18-39; 40-59; 60 anos ou mais) e grau de escolaridade (ensino médio incompleto ou menos; ensino médio completo ou mais), isto é, convidando, 
ao menos, 3 pessoas em cada um dos 12 estratos. As pessoas convidadas pelas sementes compuseram a segunda onda da cadeia de recrutamento. A cada pessoa da segunda onda, foi solicitado que ela convidasse pelo menos outras três pessoas de suas redes sociais, por meio da mensagem ao final do questionário:

Faça parte da Rede ConVid e compartilhe esta pesquisa com três ou mais convidados da sua rede social. Você pode fazer isso clicando aqui ou copiando e enviando o nosso link https://convid.fiocruz.br.

Com esse processo, a rede de convidados aumentou rapidamente; e, ao final do período de coleta das informações ( 24 de abril a 24 de maio de 2020), o tamanho total atingido de amostra foi de 45.161 pessoas.

As questões analisadas no presente estudo referem-se a adesão às medidas de restrição social, alterações nos estilos de vida (hábito de fumar; consumo de bebida alcoólica, hábito alimentare prática de atividade física) e no estado de ânimo.

A adesão às medidas de restrição social pela população brasileira foi avaliada pela pergunta: 'Durante a pandemia do novo coronavírus, com que intensidade você fez (ou ainda está fazendo) restrição do contato com as pessoas?'. As opções de respostas eram: nenhuma restrição (Não fiz nada, levei vida normal); pouca restrição (Procurei tomar cuidados, ficar à distância das pessoas, reduzir um pouco o contato, não visitar idosos, mas continuei trabalhando e saindo); restrição intensa (Fiquei em casa só saindo para compras em supermercado e farmácia); restrição total (Fiquei rigorosamente em casa, saindo só por necessidades de atendimento à saúde).

O estado de ânimo foi avaliado pelas seguintes questões: 'No período da pandemia, com que frequência você se sentiu isolado(a) dos seus familiares ou amigos próximos?'; ‘No período da pandemia, com que frequência você se sentiu triste ou deprimido(a)?'; 'No período da pandemia, com que frequência você se sentiu ansioso(a) ou nervoso(a)?'. Para todas as perguntas, as categorias de resposta eram: nunca; poucas vezes; muitas vezes; sempre.
Para o aumento de consumo de cigarros, foram avaliadas as seguintes questões: 'O sr.(a) é fumante?'; Se sim: 'Antes da pandemia, em média, quantos cigarros você costumava fumar por dia?'; e 'Durante a pandemia, em média, quantos cigarros você passou a fumar por dia?'. As opções de respostas para as duas últimas questões eram: 'Não fumava cigarros, só outros produtos'; ‘Menos que 1 por dia'; 'De 1 a 9 cigarros'; 'De 10 a 19 cigarros'; 'De 20 a 29 cigarros'; 'De 30 a 39 cigarros'; '40 ou mais cigarros'. Para avaliar a quantidade de cigarros, foi usado o ponto médio da categoria de resposta e calculada a diferença entre os dois momentos. Considerou-se aumento no consumo de cigarros quando a diferença foi positiva.

O aumento do consumo de bebida alcoólica foi avaliado pela questão: 'Durante a pandemia: a) continuei bebendo com a mesma frequência; b) estou bebendo mais do que costumava; c) estou bebendo menos do que costumava; e d) tinha parado de beber, mas comecei a beber novamente'. O aumento no consumo de álcool foi considerado pelas respostas (b, d).

Para os hábitos alimentares, foi utilizada a indicação do consumo de alimentos saudáveis (hortaliças, frutas) e não saudáveis [alimentos congelados (pizza congelada ou lasanha congelada ou outro prato pronto congelado) e salgadinhos (de pacote)] pelas questões: 'Antes da pandemia, com que frequência você comia esses alimentos?'; e 'Durante a pandemia, com que frequência você passou a comer esses alimentos?'. Para frutas e hortaliças, foi considerado como consumo regular cinco ou mais dias da semana; $\mathrm{e}$ para os congelados e salgadinhos, foi considerado como inadequado o consumo em dois ou mais dias na semana ${ }^{23}$.

A prática de atividade física foi avaliada pelas seguintes questões: 'Antes da pandemia, quantos dias por semana você praticava algum tipo de exercício físico ou esporte e quanto tempo durava essa atividade?'; 'Durante a pandemia, quantos dias por semana você pratica ou praticava exercício físico ou esporte e quanto tempo dura/durava essa atividade?'. Foram considerados suficientemente ativos os indivíduos que reportaram, pelo menos, 150 minutos semanais de atividade física ${ }^{24}$. 
Foram calculados as prevalências e os Intervalos de Confiança de 95\% (IC95\%) das variáveis estudadas. Diferenças significativas foram identificadas por meio da não sobreposição dos IC95\% das prevalências em questão.

Uma vez que a amostragem por redes não é probabilística, para obter uma amostra representativa da população, de acordo com a localização geográfica e com as características sociodemográficas, foram realizadas ponderações calculadas por procedimentos de pós-estratificação ${ }^{25}$ por: Unidade da Federação (UF), capital/restante da UF, sexo, faixa de idade (18-29; 30-39; 40-49; 50-59; 60 anos ou mais), grau de escolaridade (superior incompleto; superior completo) e raça/ cor da pele a partir estimativas populacionais da Pesquisa Nacional de Amostra por Domicílios, 2019 (PNAD-2019) do Instituto Brasileiro de Geografia e Estatística (IBGE) ${ }^{26}$. Para verificar a representação da população brasileira na amostra obtida na ConVid, as distribuições amostrais das características sociodemográficas foram comparadas com as da PNAD-201926.

O processamento das informações foi realizado no Software for Statistics and Data Science (Stata) versão 14, empregando o módulo Survey que considera os pesos de pós-estratificação.

O presente estudo constitui uma parceria entre pesquisadores da Universidade Federal de Minas Gerais, Fundação Oswaldo Cruz e Universidade Estadual de Campinas. Os participantes preencheram o Termo de Consentimento Livre e Esclarecido, e todas as respostas foram anônimas e sem qualquer tipo de identificação dos participantes. A pesquisa foi aprovada pela Comissão Nacional de Ética em Pesquisa (Número do Parecer: 3.980.277).

\section{Resultados}

Foram avaliados 45.161 indivíduos, sendo $53,6 \%$ do sexo feminino; $45,7 \%$ da população estudada tinha entre 18 e 39 anos; $34,0 \%$, entre 40 e 59 anos; e $20,3 \%, 60$ ou mais anos de idade. No que se refere à escolaridade, $72,4 \%$ dos participantes tinham ensino médio completo; $11,1 \%$, ensino fundamental incompleto; e 16,5\%, ensino superior completo ou mais.

Em relação à intensidade do distanciamento social durante o período do estudo, apenas 1,5\% (IC95\% 1,20 - 1,98) levou vida normal, sem nenhuma restrição social; e 15,1\% (IC95\% $14,1-16,1)$ ficaram rigorosamente em casa. As mulheres fizeram maior restrição total que os homens (gráfico 1).

Gráfico 1. Intensidade do distanciamento social durante a pandemia de Covid-19 segundo sexo. Brasil, 2020

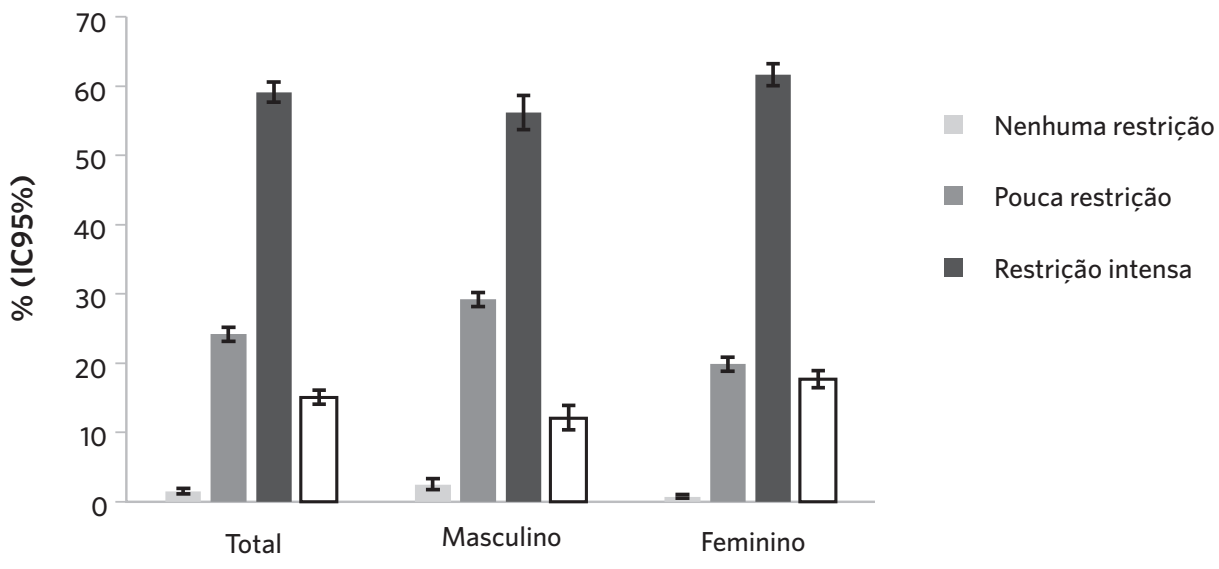


Em relação ao estado de ânimo, o estudo revelou que 41,2\% (gráfico $2 A$ ), 41,3\% (gráfico $2 B$ ) e $35,2 \%$ (gráfico $2 C$ ) dos brasileiros se sentiram isolados, ansiosos, e tristes ou deprimidos por muitas vezes, respectivamente, sendo as prevalências maiores nas mulheres comparadas aos homens.

Gráfico 2. Frequência com que se sentiu isolado (2A), ansioso (2B), e triste ou deprimido (2C) durante a pandemia de Covid-19 segundo sexo. Brasil, 2020
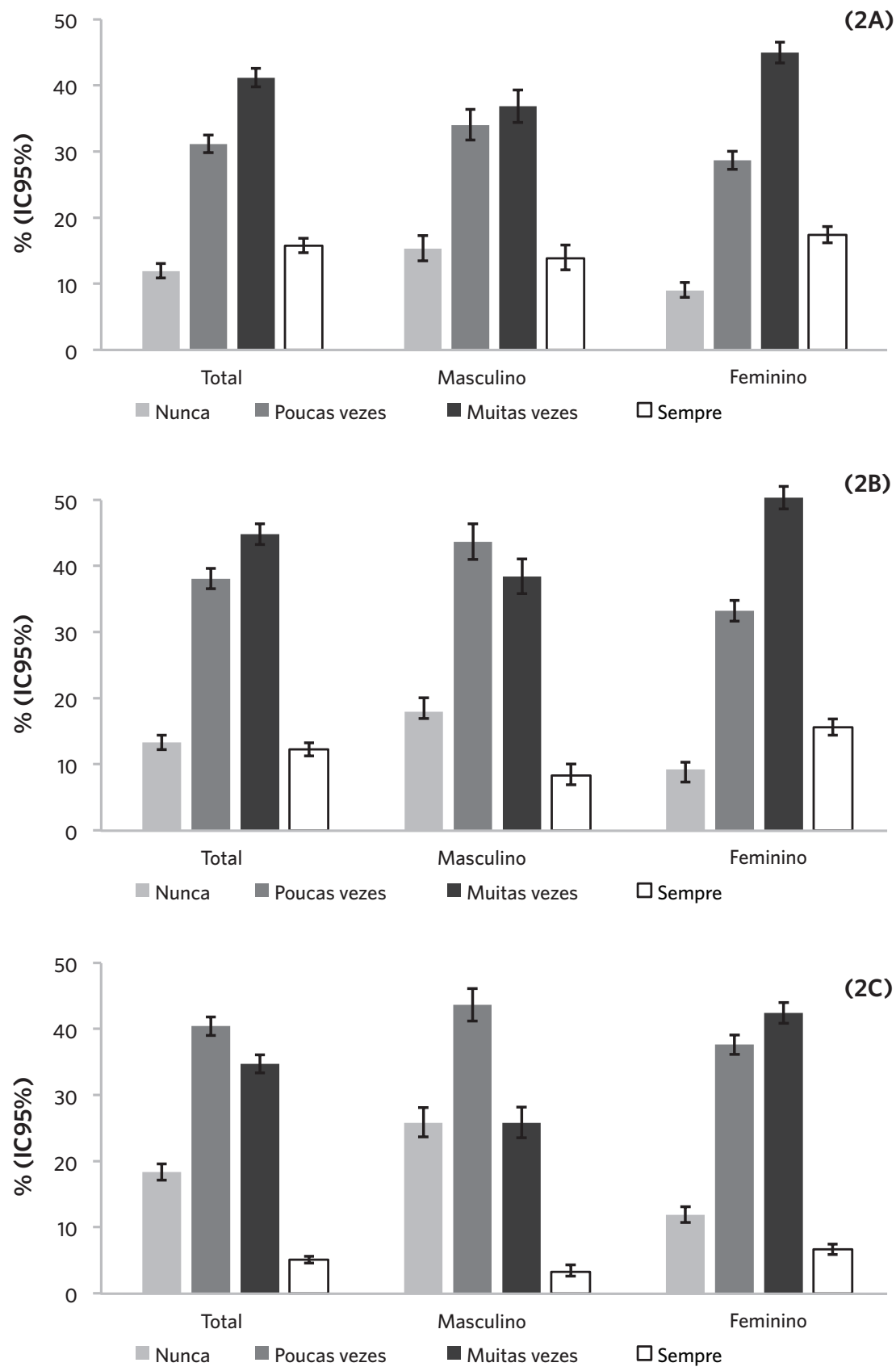

Fonte: Elaboração própria. 
O aumento de consumo de bebidas alcoólicas durante o período do estudo foi observado em $17,6 \%$ (IC95\% 16,4 - 19,0) dos respondentes, sem diferenças segundo sexo. $\mathrm{O}$ aumento foi maior em adultos com 18 a 39 anos $(21,4 \%$ IC95\% 19,4 - 23,6) e entre aqueles com maior nível de escolaridade (26,0\% IC95\% 25,2 $26,8)$. Houve aumento do uso do tabaco em
$34 \%$ dos fumantes brasileiros. As prevalências foram maiores entre as mulheres (38,4\% IC95\% 33,7 - 43,3), entre aqueles com 18 a 39 $\operatorname{anos}(37,2 \%$ IC95\% 31,0 - 43,9) e com ensino fundamental completo ou menos $(44,4 \%$ IC95\% 32,7 - 56,7), no entanto, não houve diferenças estatisticamente significativas entre os grupos (tabela 1).

Tabela 1. Aumento do consumo de bebida alcoólica e tabaco durante a pandemia de Covid-19 segundo sexo, faixa etária e escolaridade. Brasil, 2020

\begin{tabular}{lrr}
\hline Variáveis & Álcool & Tabaco \\
\hline Total & \% (IC95\%) & \% (IC95\%) \\
\cline { 2 - 3 } Sexo & $\mathbf{1 7 , 6 ( 1 6 , 4 - 1 9 , 0 )}$ & $\mathbf{3 4 , 0}(\mathbf{3 0 , 4 - 3 7 , 9 )}$ \\
Masculino & & \\
Feminino & $18,1(16,1-20,4)$ & $30,2(24,8-36,1)$ \\
Faixa etária & $17,1(15,9-18,5)$ & $38,4(33,7-43,3)$ \\
18 a 39 anos & & \\
40 a 59 anos & $21,4(19,4-23,6)$ & $37,2(31,0-43,9)$ \\
60 anos ou mais & $16,1(14,33-18,1)$ & $31,2(26,3-36,6)$ \\
Escolaridade (anos de estudo) & $11,2(8,8-14,2)$ & $33,0(25,1-41,9)$ \\
Ensino fundamental completo ou menos & & \\
Ensino médio completo & $11,0(7,8-15,26)$ & $44,4(32,7-56,7)$ \\
Ensino superior completo ou mais & $16,5(14,87-18,22)$ & $32,2(27,9-36,8)$ \\
\hline
\end{tabular}

Fonte: Elaboração própria.

No que se refere à prática de atividade física, observa-se diminuição da prática, sendo que antes da pandemia, 30,1\% (IC95\% 28,9 - 31,5) dos adultos praticavam atividade física suficiente, reduzindo para 12,0\% (IC95\% 11,1 -12,9). Entre os homens, a prevalência era de $33,1 \%$ (IC95\% 30,7 - 35,5), passando para 14,0\% (IC95\% 12,4 - 15,8). Essa diminuição também ocorreu entre as mulheres, com prevalência de 27,6\% (IC95\% 26,2 - 29,0) antes da pandemia; e, durante, de 10,3\% (IC95\% 9,4 - 11,2). Para todas as faixas etárias, houve redução da prática de atividade física, com menor percentual entre os mais jovens, passando de $31,9 \%$ (IC95\% 29,9 - 33,9) para 10,8\% (IC95\% 9,7 - 12,0). Entre os níveis de escolaridade, antes da pandemia, a maior prevalência era entre os indivíduos com ensino superior completo ou mais, (39,1\% IC95\% 38,4 - 39,8), reduzindo para 14,9\% (IC95\% 14, 5 - 15,4) (tabela 2). 
Tabela 2. Prática de atividade física antes e durante a pandemia de Covid-19 segundo sexo, faixa etária e escolaridade. Brasil, 2020

\begin{tabular}{|c|c|c|}
\hline \multirow[t]{2}{*}{ Variáveis } & $\begin{array}{r}\text { Atividade física antes } \\
\text { da pandemia }\end{array}$ & $\begin{array}{r}\text { Atividade física } \\
\text { durante a pandemia }\end{array}$ \\
\hline & $\%($ IC95\%) & $\%(I C 95 \%)$ \\
\hline Total & $30,1(28,8-31,5)$ & $12,0(11,1-12,9)$ \\
\hline \multicolumn{3}{|l|}{ Sexo } \\
\hline Masculino & $33,1(30,7-35,5)$ & $14,0(12,4-15,8)$ \\
\hline Feminino & $27,6(26,2-29,0)$ & $10,3(9,4-11,2)$ \\
\hline \multicolumn{3}{|l|}{ Faixa etária } \\
\hline 18 a 39 anos & $31,9(29,9-33,9)$ & $10,8(9,7-12,0)$ \\
\hline 40 a 59 anos & $27,6(25,7-29,7)$ & $12,4(10,9-14,0)$ \\
\hline 60 anos ou mais & $30,4(27,2-33,8)$ & $14,2(12,0-16,9)$ \\
\hline \multicolumn{3}{|l|}{ Escolaridade (anos de estudo) } \\
\hline Ensino fundamental completo ou menos & $23,2(18,5-28,7)$ & $10,81(8,1-14,3)$ \\
\hline Ensino médio completo & $29,1(27,5-30,8)$ & $11,5(10,4-12,7)$ \\
\hline Ensino superior completo ou mais & $39,1(38,4-39,8)$ & $14,9(14,5-15,4)$ \\
\hline
\end{tabular}

Fonte: Elaboração própria.

O consumo regular de hortaliças reduziu de $37,3 \%$ (IC95\% 35,9 - 38,6) para 33,0\% (IC95\% 31,7 - 34,3), e o de frutas não alterou durante o período do estudo. Por outro lado, o consumo de alimentos não saudáveis aumentou nesse período: o consumo de congelados passou de 10,0\% (IC95\% 9,0 - 11,2) para 14,6\% (IC95\% 13,5 - 15,9); e o de salgadinho, de 9,5\% (IC95\% 8,6 - 10,5) para 13,2\% (IC95\% 12,2 14,4). Estratificado por sexo, o mesmo padrão de mudanças foi observado, com aumento do consumo de salgadinhos e congelados em ambos sexos e redução do consumo de hortaliças e manutenção de frutas. As mulheres apresentaram maiores prevalências de consumo regular de frutas e hortaliças antes e depois da pandemia e menores prevalências de consumo de congelados. Em relação às faixas etárias e à escolaridade, o consumo desses alimentos seguiu padrões semelhantes ao total da amostra estudada; contudo, entre pessoas de 40 a 50 anos e 60 anos ou mais, não foi observado aumento significativo do consumo de alimentos não saudáveis durante a pandemia. Apenas entre 60 anos ou mais o consumo de hortaliças se manteve inalterado antes e durante a pandemia. Entre aqueles com ensino fundamental completo ou menos, as variações não foram significativas. Ocorreu aumento no consumo de congelados e salgadinhos entre a população com ensino médio completo. Entre a população com ensino superior ou mais, houve redução significativa do consumo regular de hortaliças de 48,8\% (IC95\% 48,1 - 49,5) para 40,9\% (IC95\% 40,3 - 41,6), e de frutas de $40,8 \%$ (IC95\% 40,1 - 41,4) para 38,1\% (IC95\% 37,5 - 38,8); e aumento significativo do consumo de congelados de $8,5 \%$ (IC95\% $8,1-9,0)$ para $16,4 \%$ (IC95\% 15,8 - 16,9), e de salgadinhos de 6,5\% (IC95\% 6,2 - 6,9) para 14,4\% (IC95\% 13,9 - 14,9) (tabela 3). 
Tabela 3. Consumo de alimentos saudáveis e não saudáveis antes e durante a pandemia de Covid-19 segundo sexo, faixa etária e escolaridade. Brasil, 2020

\begin{tabular}{|c|c|c|}
\hline \multirow[t]{2}{*}{ Variáveis } & $\begin{array}{r}\text { Alimentação antes da } \\
\text { pandemia }\end{array}$ & $\begin{array}{r}\text { Alimentação durante } \\
\text { a pandemia }\end{array}$ \\
\hline & Sim & Sim \\
\hline Total & $\%(I C 95 \%)$ & $\%($ IC95\%) \\
\hline Consumo regular de hortaliças & $37,3(35,9-38,6)$ & $33,01(31,7-34,3)$ \\
\hline Consumo regular de frutas & $32,8(31,5-34,2)$ & $31,91(30,6-33,3)$ \\
\hline Congelados & $10(9,0-11,2)$ & $14,6(13,5-15,9)$ \\
\hline Salgadinhos & $9,5(8,6-10,5)$ & $13,2(12,2-14,4)$ \\
\hline \multicolumn{3}{|l|}{ Masculino } \\
\hline Consumo regular de hortaliças & $33,3(31,1-35,6)$ & $28,6(26,5-30,8)$ \\
\hline Consumo regular de frutas & $28,3(26,1-30,6)$ & $27,1(24,9-29,4)$ \\
\hline Congelados & $12,4(10,4-14,7)$ & $17,0(14,8-19,5)$ \\
\hline Salgadinhos & $10,0(8,4-11,9)$ & $14,2(12,3-16,4)$ \\
\hline \multicolumn{3}{|l|}{ Feminino } \\
\hline Consumo regular de hortaliças & $40,7(39,2-42,2)$ & $36,8(35,4-38,3)$ \\
\hline Consumo regular de frutas & $36,8(35,3-38,3)$ & $36,1(34,6-37,7)$ \\
\hline Congelados & $7,9(7,1-8,8)$ & $12,6(11,7-13,5)$ \\
\hline Salgadinhos & $9,0(8,2-9,9)$ & $12,4(11,6-13,3)$ \\
\hline \multicolumn{3}{|l|}{ Faixa etária } \\
\hline \multicolumn{3}{|l|}{18 a 39 anos } \\
\hline Consumo regular de hortaliças & $30,8(28,9-32,7)$ & $27,0(25,3-28,8)$ \\
\hline Consumo regular de frutas & $23,2(21,5-25,1)$ & $22,0(20,2-23,9)$ \\
\hline Congelados & $12,5(10,7-14,6)$ & $19,5(17,6-21,5)$ \\
\hline Salgadinhos & $13,6(12,1-15,2)$ & $19,2(17,5-21,1)$ \\
\hline \multicolumn{3}{|l|}{40 a 59 anos } \\
\hline Consumo regular de hortaliças & $40,7(38,5-42,9)$ & $35,5(33,4-37,7)$ \\
\hline Consumo regular de frutas & $34,0(31,8-36,2)$ & $33,7(31,5-35,9)$ \\
\hline Congelados & $8,7(7,2-10,5)$ & $12,0(10,3-13,8)$ \\
\hline Salgadinhos & $7,4(5,9-9,1)$ & $10,1(8,6-11,9)$ \\
\hline \multicolumn{3}{|l|}{60 anos ou mais } \\
\hline Consumo regular de hortaliças & $46,2(42,8-49,5)$ & $42,5(39,2-45,8)$ \\
\hline
\end{tabular}

\section{Discussão}

Esta é a primeira pesquisa realizada com uma amostra de adultos brasileiros durante o início da pandemia de Covid-19, e aponta que a maior parte da população brasileira aderiu ao distanciamento social, saindo apenas para ir ao supermercado e farmácia ou ficaram rigorosamente em casa. Em contrapartida, o distanciamento social contribuiu para um impacto negativo na saúde mental e nos estilos de vida dos brasileiros, com aumento do sentimento de ansiedade, isolamento, tristeza ou depressão. Além disso, observou-se aumento do uso de 
bebidas alcoólicas e tabaco, redução da prática de atividade física e aumento do consumo de alimentos não saudáveis.

O estudo aponta a importante adesão dos brasileiros ao isolamento social no período do estudo. A adesão às medidas de distanciamento tem impacto significativo no achatamento da curva e dos casos, bem como na redução da mortalidade $\mathbf{9 1 0 , 2 7}$. Medidas de distanciamento social também foram utilizadas para o controle das epidemias anteriores, como da Síndrome Respiratória Aguda Grave (SARS), da Síndrome Respiratória do Médio Oriente (MERS) e durante o surto de Ebola de 201428. No Brasil, estudo realizado em Sergipe mostrou uma relação inversa e estatisticamente significante entre os índices de isolamento social e o número de casos novos de Covid-1929.

Cabe salientar que as medidas de distanciamento social podem ocasionar implicações psicológicas, como ansiedade, tristeza e depressão. $\mathrm{O}$ medo de ser infectado por um vírus potencialmente fatal ${ }^{5}$, somado às preocupações com as perdas financeiras, pode afetar o bem-estar psicológico de muitas pessoas que poderão desenvolver sintomas de depressão, ansiedade e estresse ${ }^{30}$. Os sentimentos de ansiedade, estresse também foram descritos em estudos na China, na Espanha e na Colômbia 11,12,31. Nesse sentido, além das medidas para o enfrentamento e contenção da pandemia, é preciso garantir à população uma assistência apropriada em saúde mental, com ações que minimizem o sofrimento mental ao longo desse período de crise. Ademais, é importante propor estratégias de promoção da saúde mental e de atenção psicossocial em curto, médio e longo prazo, na tentativa de reduzir ou prevenir problemas psiquiátricos e psicológicos 32,33 .

O distanciamento social relacionado com a pandemia tem sido considerado como um evento estressante, e um dos efeitos da permanência prolongada em casa também tem sido o aumento do consumo de álcool e tabaco ${ }^{34,35}$.

Alguns potenciais mecanismos que acompanham o impacto da Covid-19 e o uso de álcool são descritos na literatura. O primeiro mecanismo sugere que o aumento do sofrimento psicológico desencadeado pelo isolamento social, as dificuldades financeiras e a incerteza sobre o futuro durante e após a pandemia podem piorar os padrões de consumo de álcool ${ }^{36}$. Outro mecanismo aborda situações que podem culminar na diminuição do consumo e tem relação com a disponibilidade física, acessibilidade financeira que pode ser afetada pela redução da renda e orçamentos mais apertados, e políticas regulatórias de controle do álcool ${ }^{36,37}$. Destaca-se que o consumo de álcool pode potencialmente piorar o curso clínico da infecção por Covid-19. A exposição crônica ao álcool tem um efeito complexo e adverso na resposta do hospedeiro ${ }^{38}$, incluindo os mecanismos de imunidade inata e adaptativa, e é conhecida por aumentar a suscetibilidade à infecção viral ${ }^{39}$. No Reino Unido, durante o período de confinamento, as vendas de bebidas alcoólicas aumentaram $67 \%{ }^{40}$. Uma pesquisa realizada na Austrália identificou que aproximadamente um quarto dos participantes $(22,3 \%)$ relataram mudanças negativas de padrões do uso de álcool com consumo em quatro ou mais ocasiões por semana ${ }^{35}$. Outro estudo, realizado na China, identificou maior consumo de bebida alcoólica entre homens e em indivíduos com idade entre 21 e 40 anos $^{\mathbf{1 2}}$.

Adicionalmente, o aumento do estresse de uma doença potencialmente fatal, a possibilidade de perda de emprego, o sentimento de insegurança, o confinamento e o tédio podem aumentar o desejo de fumar ${ }^{41-43}$. Sabe-se que o tabagismo está relacionado com transtornos mentais e comorbidades psiquiátricas, tais como a depressão, o transtorno de humor, a ansiedade e outros transtornos mentais ${ }^{44,45}$, que são comorbidades comuns durante a pandemia e que podem ser agravadas em período de distanciamento social ${ }^{\mathbf{4 6}, 47}$. No intuito de enfrentar o tabagismo durante a pandemia da Covid-19, a África do Sul proibiu a venda de produtos de tabaco e nicotina, designando-os como como bens não essenciais, o que deve ser compreendido como uma tentativa 
dupla de proteger a população da Covid-19 e do ônus evitável do tabaco ${ }^{48}$.

Para deter o consumo de tabaco e de álcool, a OMS preconiza as seguintes medidas: a proibição da propaganda, o aumento de taxação e preços, a legislação referente ao ambiente livre de tabaco, o aumento das advertências sanitárias, a restrição de pontos de venda, além da fiscalização na aplicação das medidas ${ }^{49}$. Esses pontos levantam a importância de avançar na adoção de novas medidas regulatórias para tabaco e álcool21. Ressaltam-se ainda as medidas educativas, como a divulgação de informação sobre os malefícios no consumo dessas substâncias, o que poderá contribuir para aumentar o conhecimento da população e colaborar para a mudança de comportamentos individuais, além de manter esses assuntos na agenda social e política ${ }^{50}$.

A redução da atividade física e o aumento de estilos sedentários são prejudiciais para a saúde e afetam negativamente a qualidade de vida ${ }^{51}$. O aumento do comportamento sedentário, como tempo em frente à TV, tablet e computadores, tem efeitos prejudiciais na função e na estrutura cardiovascular ${ }^{15}$, bem como na saúde mental17 e nas mortes prematuras $^{52}$. As recomendações de saúde pública para evitar a disseminação da Covid-19 têm o potencial de reduzir a atividade física diária. Assim, medidas são necessárias para orientar a população sobre estratégias de manutenção de hábitos ativos durante o período de quarentena, especialmente para os grupos populacionais mais afetados ${ }^{53}$.

O distanciamento social também impacta nos hábitos alimentares. Ficar em quarentena pode causar tédio, o que está associado a um maior consumo alimentar. Ter muito acesso às informações referentes à Covid-19 pode provocar estresse nos indivíduos, levando também ao consumo excessivo de alimentos, principalmente os ricos em açúcares, chamados como 'comida afetiva' ${ }^{\text {'6, }}$, pois estes estimulam a produção de serotonina, causando um efeito positivo no humor. Nos Estados Unidos da América, no início da pandemia, também foi identificado um aumento no volume de compras em supermercados de alimentos ultraprocessados e com alta densidade energética, como batata frita, pipoca, chocolate e sorvete $^{16-48}$. Ademais, esses alimentos são mais fáceis de conservação, tornando-se mais acessíveis, já que sair para realizar abastecimentos diários torna-se difícil durante a pandemia.

Diferentemente do Brasil, população em países como a Itália não mudou tanto seus hábitos alimentares. Estudo Italiano investigou o consumo alimentar durante a pandemia em cerca de 3.533 participantes e comparou os desfechos de aumentar ou reduzir o consumo de determinados alimentos. Os resultados apontaram que o consumo de frutas e verduras não se alterou (cerca de 37\%) durante a pandemia e, ao contrário do Brasil, houve redução no consumo de alimentos ultraprocessados, ou junk foods ${ }^{47}$.

Entre as limitações do estudo atual, discute-se a coleta de dados pela internet, que pode não atingir todos os estratos populacionais, considerando que nem todos têm acesso a esse meio de comunicação. Esse fato pode levar a sub ou superestimação da proporção dos indicadores. Essa limitação foi minimizada devido ao grande número amostral e à calibração da amostra com os dados da PNAD contínua de 2019. Outra limitação refere-se ao fato de a coleta de dados ter sido realizada no início da pandemia (abril e maio), o que reflete um momento específico, sujeito a mudanças ao longo do tempo. Adicionalmente, alguns indicadores (consumo de álcool e tabaco) não foram avaliados antes da pandemia, medindo-se apenas o aumento da utilização durante o período de estudo.

Cabe destacar, ainda, que a coleta de dados via internet e o processo de amostragem em cadeia são o único método, eticamente, plausível no âmbito da pandemia, além do baixo custo relacionado com a operacionalização. As pesquisas on-line surgem como um método promissor para avaliar e rastrear conhecimentos, comportamentos, estilos de vida e percepções durante surtos de doenças infecciosas em rápida evolução. 


\section{Considerações finais}

O distanciamento social constitui-se medida essencial de proteção à vida, de redução de exposição, diminuindo casos e, consequentemente, a morbimortalidade. Entretanto, o estudo apontou que essa medida pode resultar em consequências psicossociais e no estilo de vida da população. Evidenciou-se frequência elevada de sentimentos de isolamento, ansiedade e tristeza no período do estudo, bem como aumento do uso de bebida alcoólica e cigarros, redução da prática de atividade física e aumento do consumo de alimentos não saudáveis, como congelados e salgadinhos. Esses achados são preocupantes e podem resultar em danos à saúde, como alterações no peso corporal, aumento de DCNT e implicações psicológicas.

Esses resultados podem apoiar profissionais de saúde e gestores na priorização de estratégias de promoção de saúde durante e após a pandemia. Torna-se fundamental a continuidade das ações da atenção primária à saúde, no acompanhamento aos usuários, com atenção aos portadores de DCNT, reforçando os comportamentos saudáveis e a continuidade dos cuidados. Atenção à saúde mental deve ser prioridade. Mensagens educativas sobre hábitos saudáveis para toda a população devem ser desencadeadas pelos governos, bem como ações intersetoriais de promoção à saúde.

\section{Colaboradores}

Malta DC (0000-0002-8214-5734)*, Gomes CS (0000-0001-6586-4561)*, Szwarcwald CL (00000002-7798-2095)*, Silva AG (0000-0003-25875658)*, Prates EJS (0000-0002-5049-186X)* e Azevedo LO (0000-0002-4876-5948)* contribuíram igualmente para concepção, planejamento, análise e interpretação dos dados; revisão crítica do conteúdo; e aprovação da versão final do manuscrito. Barros MBA (0000-0003-3974-195X)*, Lima MG (0000-0001-6996-0745)*e Damacena GN (0000-0002-7059-3353)* contribuíram igualmente para a análise e a interpretação dos dados; revisão crítica do conteúdo; e aprovação da versão final do manuscrito. Machado IE (0000-00024678-2074)*, Romero DE (0000-0002-26439797)*, Werneck AO (0000-0002-9166-4376)*e Pina MF (0000-0002-1521-7865)* contribuíram igualmente para revisão crítica do manuscrito e aprovação da versão final. Souza Júnior PRB (0000-0002-8142-4790)* contribuiu para aprovação final da versão a ser publicada e se responsabilizou por todos os aspectos do trabalho, incluindo a garantia de sua precisão e integridade. Silva DRP (0000-0003-3995-4795)* contribuiu para o delineamento do estudo e aquisição dos dados do trabalho; revisão crítica do material; aprovação final da versão a ser publicada; concordância em ser responsável por todos os aspectos do trabalho, no sentido de garantir que as questões relacionadas à exatidão ou à integridade de qualquer parte da obra sejam devidamente investigadas e resolvidas. 


\section{Referências}

1. Organização Mundial da Saúde. WHO Director-General's opening remarks at the media briefing on COVID-19-11 March 2020 [internet]. Genebra: OMS; 2020. [acesso em 2020 jun 17]. Disponível em: https://www.who.int/dg/speeches/detail/who-director-general-s-opening-remarks-at-the-media-briefing-on-covid-19---11-march-2020.

2. Croda JHR, Garcia LP. Resposta imediata da Vigilância em Saúde à Pandemia da COVID-19. Epidemiol Serv Saúde 2020; 29(1):e2020002.

3. Brasil. Ministério da Saúde, Secretaria de Vigilância em Saúde. Boletim Epidemiológico Especial COE-COVID19 [internet]. 2020. [acesso em 2020 jun 17]. Disponível em: https:/www.saude.gov.br/images/pdf/2020/ May/29/2020-05-25---BEE17---Boletim-do-COE.pdf.

4. Organização Mundial da Saúde. Coronavirus disease (COVID-19) pandemic [internet]. Genebra: OMS; 2020. [acesso em 2020 jun 17]. Disponível em: https:// www.who.int/emergencies/diseases/novel-coronavirus-2019.

5. Brooks SK, Webster RK, Smith LE, et al. The psychological impact of quarantine and how to reduce it: rapid review of the evidence. Lancet. 2020; 395(10227):912-920.

6. Carlson CJ. From PREDICT to prevention, one pandemic later. The Lancet Microbe. 2020; 1(1):e6-e7.

7. Belo Horizonte. Prefeitura Municipal. Decreto $n^{\circ}$ 17.297, de 17 de março de 2020. Declara situação anormal, caracterizada como Situação de Emergência em Saúde Pública, no Município de Belo Horizonte em razão da necessidade de ações para conter a propagação de infecção viral, bem como de preservar a saúde da população contra o Coronavírus - COVID-19 [internet]. 2020. [acesso em 2020 jun 17]. Disponível em: http://portal6.pbh.gov.br/dom/iniciaEdicao.do? method=DetalheArtigo\&amp;pk=1226967.

8. Maranhão. Governo do Estado. Decreto $\mathrm{n}^{\circ} 35.678$ de 22 de março de 2020. Altera o Decreto nº 35.677 , de 21 de março de 2020, que estabelece medidas de prevenção do contágio e de combate à propagação da transmissão da COVID- 19, infecção humana causada pelo Coronavírus (SARS-CoV-2) [internet]. 2020. [acesso em 2020 jun 17]. Disponível em: https://sedihpop.ma.gov.br/files/2020/03/DECRETO-N-35.678DE-22-DE-MARC\%CC\%A7O-DE-2020.-VF.pdf.pdf.

9. Nussbaumer-Streit B, Mayr V, Dobrescu AI, et al. Quarantine alone or in combination with other public health measures to control COVID 19: a rapid review. Cochrane Database of Syst Rev. 2020; 4(4):CD013574.

10. Aquino E, Silveira IH, Pescarini J, et al. Medidas de distanciamento social no controle da pandemia de COVID-19: Potenciais impactos e desafios no Brasil. Ciênc. Saúde Colet. 2020; 25(supl1):2423-2446.

11. Pedrozo-Pupo JC, Pedrozo-Cortes MJ, Campo-Arias A. Perceived stress associated with COVID-19 epidemic in Colombia: an online survey. Cad. Saúde Pública 2020; 36(5):e00090520.

12. Ahmed MZ, Ahmed O, Aibao Z, et al. Epidemic of COVID-19 in China and associated Psychological Problems. Asian J Psychiatr 2020; 51:102092.

13. Wang C, Pan R, Wan X, et al. A longitudinal study on the mental health of general population during the COVID-19 epidemic in China. Brain Behav Immun. 2020; S0889-1591(20):30511-0.

14. García-Álvarez L, Fuente-Tomás L, Sáiz PA, et al. Will changes in alcohol and tobacco use be seen during the COVID-19 lockdown? Adicciones. 2020; 32(2):85-89.

15. Peçanha T, Goessler KF, Roschel H, et al. Social isolation during the COVID-19 pandemic can increase physical inactivity and the global burden of cardiovascular disease. Am J Physiol Heart Circ Physiol. 2020; 318(6):1441-1446.

16. Bhutani S, Cooper JA. COVID-19 related home confinement in adults: weight gain risks and opportunities. Obesity. 2020; 28(9):1576-1577. 
17. Werneck GL, Carvalho MS. A pandemia de COVID-19 no Brasil: crônica de uma crise sanitária anunciada. Cad. Saúde Pública. 2020; 36(5):e00068820.

18. Doniec K, Dall'Alba R, King L. Austerity threatens universal health coverage in Brazil. Lancet. 2016; 388(10047):867-868.

19. Massuda A, Hone T, Leles FAG, et al. The Brazilian health system at crossroads: progress, crisis and resilience. BMJ Glob Health. 2018; 3(4):e000829.

20. Castro MC, Massuda A, Almeida G, et al. Brazil's unified health system: the first 30 years and prospects for the future. Lancet. 2019; 394(10195):345-356.

21. Malta DC, Duncan BB, Barros MBA, et al. Medidas de austeridade fiscal comprometem metas de controle de doenças não transmissíveis no Brasil. Ciênc. Saúde Colet. 2018; 23(10):3115-3122.

22. Costa BRL. Bola de neve virtual: o uso das redes sociais virtuais no processo de coleta de dados de uma pesquisa científica. Rev. Inter. Gest. Soc. 2018; 7(1):15-37.

23. Organização Mundial da Saúde. Diet, nutrition and the prevention of chronic diseases: report of a joint WHO/FAO expert consultation. Genebra: OMS; 2003.

24. Organização Mundial da Saúde. Global recommendations on physical activity for health. Genebra: OMS; 2011.

25. Szwarcwald CL, Damacena GN. Amostras complexas em inquéritos: planejamento e implicações na análise estatística de dados. Rev Bras Epidemiologia. 2008; 11(supl1):38-45.

26. Instituto Brasileiro de Geografia e Estatística. Pesquisa Nacional por Amostra de Domicílios (PNAD) 2019[internet]. Rio de Janeiro: IBGE; 2019. [acesso em 2020 jun 17]. Disponível em: https://biblioteca. ibge.gov.br/visualizacao/livros/liv101651_notas_tecnicas.pdf.

27. Szwarcwald, CL, Souza Júnior PRB, Malta DC, et al. Adesão às medidas de restrição de contato físi- co e disseminação da covid-19 no Brasil. Epidemiol. Serv. Saúde. No prelo 2020.

28. Conti AA. Historical and methodological highlights of quarantine measures: from ancient plague epidemics to current coronavirus disease (COVID-19) pandemic. Acta Biomed. 2020; 91(2):226-229.

29. Martins-Filho PR. Relação de Causa-Efeito entre Isolamento Social e Casos de COVID-19 em Sergipe [internet]. Aracaju: Universidade Federal de Sergipe; 2020. (Nota Técnica LPI-UFS. No 02-2020). [acesso em 2020 jun 17]. Disponível em: https://todoscontraocorona.net.br/wp-content/uploads/2020/05/Nota_ Tecnica_COVID19_Sergipe_isolamento_RG.pdf.

30. Schmidt B, Crepaldi MA, Bolze DAS, et al. Saúde mental e intervenções psicológicas diante da pandemia do novo coronavírus (COVID-19). Estud psicol. 2020; 37:e200063.

31. Ozamiz-Etxebarria N, Dosil-Santamaria M, Picaza-Gorrochategui M, et al. Niveles de estrés, ansiedad y depresión en la primera fase del brote del COVID-19 en una muestra recogida en el norte de España. Cad. Saúde Pública. 2020; 36(4):e00054.

32. Faro A, Bahiano MA, Nakano TC, et al. COVID-19 e saúde mental: a emergência do cuidado. Estud. Psic. 2020; (37):e200074.

33. Fundação Oswaldo Cruz. Saúde Mental e Atenção Psicossocial na Pandemia Covid-19 - A quarentena na Covid-19 - orientações e estratégias de cuidado [internet]. Brasília, DF: Fiocruz; 2020. [acesso em 2020 jun 17]. Disponível em: https://www.fiocruzbrasilia.fiocruz.br/wp-content/uploads/2020/04/ Sa\%c3\%bade-Mental-e-Aten\%c3\%a7\%c3\%a3o-Psicossocial-na-Pandemia-Covid-19-A-quarentena-na-Covid-19-orienta\%c3\%a7\%c3\%b5es-e-estrat\%c3\%a9gias-de-cuidado.pdf.

34. Wu P, Liu X, Fang Y, et al. Alcohol abuse/dependence symptoms among hospital employees exposed to a SARS outbreak. Alcohol Alcohol. 2008; 43(6):706-712. 
35. Stanton R, To QG, Khalesi S, et al. Depression, Anxiety and Stress During COVID-19: Associations With Changes in Physical Activity, Sleep, Tobacco and Alcohol Use in Australian Adults. Int J Environ Res Public Health. 2020; 17(11):4065.

36. Rehm J, Kilian C, Ferreira-Borges C, et al. Alcohol use in times of the COVID 19: Implications for monitoring and policy. Drug Alcohol Rev. 2020; 39(4):301304.

37. Babor TF, Caetano R, Casswell S, et al. Alcohol: no ordinary commodity: research and public policy - Revised edition. Oxford: Oxford University Press; 2010.

38. Szabo G, Saha B. Alcohol's Effect on Host Defense. Alcohol Res. 2015; 37(2):159-170.

39. Sidor A, Rzymski P. Dietary Choices and Habits During COVID-19 Lockdown: Experience From Poland. Nutrients. 2020; 12(6):1657.

40. Fynlay I, Gilmore I. Covid-19 and Alcohol-A Dangerous Cocktail. BMJ. 2020; (369):m1987.

41. Gross GM, Bastian LA, Smith N, et al. Sex differences in associations between depression and posttraumatic stress disorder symptoms and tobacco use among veterans of recent conflicts. J Womens Health. 2020; 29(5):677-685.

42. Jiménez-Treviño L, Velasco A, Rodriguez-Revuelta $\mathrm{J}$, et al. Factors associated with tobacco consumption in patients with depression. Adicciones. 2019; 31(4):298-308.

43. Japuntich SJ, Gregor K, Pineles SL, et al. Deployment stress, tobacco use, and postdeployment posttraumatic stress disorder: Gender differences. Psychol Trauma 2016; 8(2):123-126.

44. Calheiros PRV, Oliveira MS, Andretta I. Comorbidades psiquiátricas no tabagismo. Aletheia. 2006; (23):65-74.
45. Malbergier A, Oliveira Jr HP. Dependência de tabaco e comorbidade psiquiátrica. Rev psiquiatr. Clín. 2005; 32(5):276-282.

46. Castro-de-Araujo LFS, Machado DB. Impact of COVID-19 on mental health in a Low and Middle-Income Country. Ciênc. Saúde Colet. 2020; 25(supll):24572460.

47. Silva DAR, Pimentel RFW, Merces MC. Covid-19 and the pandemic of fear: reflections on mental health. Rev Saúde Pública 2020; (54):46.

48. Egbe CO, Ngobese SP. COVID-19 lockdown and the tobacco product ban in South Africa. Tob Induc Dis. 2020; (18):39.

49. Organização Mundial da Saúde. WHO Best buys' and other recommended interventions for the prevention and control of noncommunicable diseases [internet]. Genebra: OMS; 2020. [acesso em 2020 jun 17]. Disponível em: https://apps.who.int/iris/bitstream/handle/10665/259232/WHO-NMH-NVI-17.9-eng.pdf.

50. Costa A, Cortes M, Duarte A, et al. A Lei do Tabaco em Portugal: análise da mancha mediática (20102013). Rev. Port. Saúde Púb. 2016; 34(1):85-91.

51. Lee IM, Shiroma EJ, Lobelo F, et al. Effect of physical inactivity on major non-communicable diseases worldwide: an analysis of burden of disease and life expectancy. Lancet. 2012; 380(9838):219-29.

52. Stamatakis E, Gale J, Bauman A, et al. Sitting time, physical activity, and risk of mortality in adults. J. Am. Coll. Cardiol. 2019; 73: 2062-72.

53. Sallis JF, Adlakha D, Oyeyemi A, et al. An international physical activity and public health research agenda to inform COVID-19 policies and practices. J Sport Health Sci. 2020; 9(4):328-34.

\footnotetext{
Recebido em 29/06/2020

Aprovado em 28/09/2020

Conflito de interesses: inexistente
}

Suporte financeiro: não houve 